\title{
FATORES CRÍTICOS DE SUCESSO DE UMA START- UP: UM ESTUDO BIBLIOMÉTRICO
}

Otto Herman Pedreira Goecking ${ }^{1}$

Vinicius Vargas ${ }^{2}$

Cristiana Fernandes De Muylder ${ }^{1}$

Carlos Gonçalves ${ }^{3}$

${ }^{1}$ UNIVERSIDADE FUMEC

${ }^{2}$ FACE / Fundação / UNIVERSIDADE FUMEC

${ }^{3}$ Stricto Senso / Instituto / UNIVERSIDADE FUMEC 


\section{FATORES CRÍTICOS DE SUCESSO DE UMA START- UP: UM ESTUDO BIBLIOMÉTRICO}

\section{RESUMO}

Nos últimos anos, o que se tem visto é um crescimento expressivo do segmento de startup, embora o índice de mortalidade deste tipo de empresa ainda seja alto. Portanto, este artigo tem como objetivo identificar os principais fatores críticos de sucesso de uma Startup. Foi realizada uma análise de conteúdo com base bibliográfica, partir de periódicos internacionais pesquisados no período compreendido entre 2014 e 2018. Os descritores utilizados foram fatores críticos de sucesso e startup. A amostra final foram 40 artigos. A partir da análise da amostra ficou evidenciado uma convergência entre os objetivos deste trabalho e os artigos pesquisados. Foi possível verificar ainda uma similaridade entre este trabalho e os artigos pesquisados em relação aos principais conceitos apresentados no referencial teórico.

PALAVRAS-CHAVE: Fatores críticos de sucesso; Startup.

\section{ABSTRACT}

In recent years, what has been seen is a significant growth of the startup segment, although the mortality rate of this type of company is still high. This article aims to identify the main critical success factors of a Startup. A content analysis was carried out with bibliographical basis, starting from international journals surveyed in the period between 2014 and 2018. The descriptors used were critical success factors and startup. The final sample was 40 articles. From the analysis of the sample it was evidenced a convergence between the objectives of this work and the articles researched. It was possible to verify a similarity between this work and the articles researched in relation to the main concepts presented in the theoretical reference.

KEYWORDS: Critical success factors; Startup.

\section{Introdução}

Desde a década de 40 Schumpeter já dizia que as organizações são determinantes para o funcionamento e desenvolvimento do sistema capitalista e que o empreendedorismo possibilita a geração de novos modelos de negócio, novas formas de produção e novos produtos. A novidade é que hoje a forma de empreender é diferente dos modelos desenvolvidos até a década de 90. Esta nova forma de empreender são as chamadas Startups, empresas criadas geralmente para atender um mercado novo, caracterizado por alta incerteza (Ries, 2012; Meyer 2012; Blank \& Dorf, 2014).

Ries (2012) diz que uma Startup é uma instituição criada para entregar um novo produto ou serviço, podendo ser reproduzido de forma repetitiva em alta escala, em um ambiente de extrema incerteza. Meyer (2012), complementa dizendo que as startups geralmente iniciam com estrutura e faturamento pequenos, no entanto, pensam grande e agregam ideias inovadoras possibilitando que seus negócios ganhem escala muito rapidamente. $\mathrm{O}$ grande desafio de 
startups é manterem-se no mercado, diante das incertezas que circundam a inovação (Ries, 2012; Blank \& Dorf, 2014).

$\mathrm{O}$ ambiente de incerteza reforça a necessidade e frequência, os empreendedores de startups precisarão tomar decisões, como, por exemplo, determinar a demanda do mercado por um produto ou serviço recém-desenvolvido, sem ter informações adequadas nem completas (Rogers, 2011).

Diante deste cenário de extrema incerteza, surgiram diversos modelos de criação e estruturação de startups, com o intuito de minimizar os riscos de mortalidade. Destacam-se os modelos de Blank \& Dorf (2014), chamado de "desenvolvimento de clientes" e o de Ries (2012) Lean startup.

De acordo com o jornal Folha de São Paulo, março de 2018, os investimentos em startup no Brasil, por meio de fundos de venture capital (capital de risco, que aplicam em companhias novatas no mercado) cresceu $207 \%$ em 2017 e atingiu o patamar recorde de US\$860 milhões (cerca de R \$2,9 bilhões). Em 2016, os investimentos haviam somado US\$279 milhões (R \$926 milhões). Os dados são de um estudo que está sendo realizado pela LAVCA (Associação Latino Americana de Fundos de Capital de Risco). Embora estes números sejam robustos e animadores, Patell (2018) aponta que a taxa de sucesso das startups é de 10\%, ou seja, de cada 10 que abrem, uma realmente prospera. Há necessidade, portanto, de se buscar mais evidências empíricas sobre o impacto do processo envolvendo a validação da ideia e implementação do modelo de negócio, na mortalidade de startups de base tecnológica.

Considerando o cenário apresentado, este artigo tem como objetivo investigar os fatores críticos de sucesso de startups de base tecnológica a partir de estudos científicos internacionais. Para tanto, foi realizada uma pesquisa a partir das bases do Google Acadêmico ${ }^{\circledR}$ e base Emerald $\AA$, em dezembro de 2018.

Justifica-se esta pesquisa, academicamente, frente a necessidade de avançar nos estudos de Liu \& Hong 2016; Geibel \& Manickam 2018; Kim; Kim \& Jeon, 2018; Kowanda; Firdaus; Pasaribu, \& Nawangsari, 2018; Prasad; Shankar \& Roy 2018; Ripsas; Schaper \& Tröger, 2018; Rhéaume, \& Tremblay 2018;Tomy; \& Pardede, 2018), que ressaltam a relevância de fatores críticos de sucesso de uma startup e o estudo de Powell (2017), que aborda os fatores críticos de insucesso deste mesmo tipo de empreendimento.

Socialmente, este estudo ainda contribui com a discussão sobre a alta taxa de mortalidade das startups e isto implica diretamente no aumento da taxa de desemprego e crescimento da economia.

\section{Referencial teórico}

Este capítulo tem o propósito de apresentar os princiapis conceitos sobre o ecossistema das startups. Iniciará abordando a criação de novos negócios e posteriormente serão apresentados as definições mais relevantes sobre startups.

Desde a década de 1950 até o dias atuais, estudos buscam indentificar os motivos que levam indivíduos a empreender, com destaque para os fatores necessidade e oportunidade (McClelland, 1972; Ummah e Gulapalan, 2012; Sivapalan \& Balasundaram, 2012) enquanto outros estudos sugerem que as motivações para empreender extrapolam o tema oportunidade e 
necessidade (Williams \& Round, 2009; Sivapalan \& Balasundaram, 2012; Ummah \& Gunapalan, 2012;).

Empreendedorismo é o processo de desenvolvimento de um modelo de negócio inovador e de criação de valor, para um grupo de pessoas, empenhando tempo e o esforço necessário, responsabilizando pelos riscos financeiros, psicológicos e sociais associados e recebendo as respectivas recompensas da satisfação financeira e pessoal (Faltin e Ripsas, 2011; Kohler 2016). Assim, empreendedores são aquelas pessoas que possuem o seguinte perfil: assumem riscos calculados, são persistentes, criativos, inovadores, possuem motivação para se destacar, são sociáveis, planejadores, detectam oportunidades e têm capacidade de liderança (Schmidt e Bohnenberger 2009; Radu, Daniel, Cristian, Irina \& Rodica, 2015).

De forma mais geral, (McCann \& Vroom, 2015; Packard, Clark, \& Klein 2017; Gielnik, Zacher \& Wang 2018) definiram o processo empreendedor como todas as funções, atividades e ações associadas à identificação de oportunidades e à criação de empresas para aproveitá-las. Uma ênfase comum entre os autores é que uma oportunidade pode ser explorada a partir das atitudes dos empreendedores e que esta oportunidade deve ser alvo de uma análise de viabilidade, por meio de um plano de negócios. Segundo estes mesmos autores, só assim a ideia inicial do empreendedor se transformará em uma oportunidade.

O processo empreendedor pode sofrer a influência de uma série de variáveis de cunho individual como motivações, habilidades e processo cognitivo e também intrapessoais, como relacionamento entre empreendedores e outras pessoas e sociais que englobam políticas governamentais, economia e condições de mercado. Esta influência confere grande complexidade ao processo, o que requer o uso de todas as ferramentas conceituais disponíveis na tentativa de entender como tudo acontece (McCann \& Vroom, 2015; Packard, Clark, \& Klein 2017; Gielnik, Zacher \& Wang 2018).

Associado ao conceito de empreendedorismo e ao processo empreendedor, nos últimos anos surgiu uma nova forma de empreender: as Startups. Startup é uma empresa temporária que tem o intuito de validar um modelo de negócios escalável, repetível e que atuará num ambiente de alta incerteza (Ries, 2012; Blank e Dorf, 2014).

Esta nova maneira de empreender adota uma perspectiva dinâmica e insere o modelo de negócio em vez do plano de negócios no foco do empreendedor (Faltin \& Ripsas, 2011). O modelo de negócio engloba um conjunto de premissas que precisam ser testadas no mercado, principalmente para validar a ideia, envolvendo um processo de iteração constante com os potenciais clientes (Maurya, 2012, Sosna et al., 2010, Blank \& Dorf 2014).

Para maximizar as possibilidades de sucesso de uma startup, surge o processo de desenvolvimento do cliente, que engloba as etapas referentes a descoberta e validação do cliente, geração de demanda e estruturação do negócio (Blank \& Dorf 2014; Maurya, 2012). Um ponto fundamental deste processo é a identificação clara de uma necessidade e lançamento de um produto/serviço que venha atendê-la. Para tanto, isto pode demandar várias iterações e consequentes feedbacks dos clientes, resultando em algumas pivotagens, até que se consiga encontrar este ponto focal (Maurya, 2012; Ries, 2012; Blank \& Dorf, 2014).

Blank e Dorf (2012), Ries (2012) e Bakator, Đorđević, Ćoćkalo, Nikolić, \& Vorkapić 2018). argumentam que empresas estabelecidas executam um modelo de negócios, enquanto startups 
procuram por um. Essa definição está no cerne da abordagem enxuta e a partir dela surge a definição de Lean Startup.

Ries (2012) declarou que o lean startup foi concebido tendo como principais pilares a metodologia customer development e a filosofia por trás da gestão ágil. Segundo o lean startup, o empreendedor deve traduzir a sua visão na forma de hipóteses refutáveis do seu modelo de negócio, realizando uma série de testes de validação por meio de mínimos produtos viáveis (ou MVPs, do inglês minimum viable products), (Maurya, 2012).

Embora este novo modelo de negócios venha crescendo de forma vertiginosa, em 2014 foi divulgada uma pesquisa pelo Startup Genome Report Extra on Premature Scaling, mostrando que de um universo de 3200 startups pesquisadas, $92 \%$ falharam nos três primeiros anos, sendo que $74 \%$ falharam devido ao escalonamento prematuro. Escalar prematuramente significa investimentos em divulgação e estruturação do negócio antes de encontrar um modelo de negócios eficaz, com a ideia validada (Behrens, 2015).

Para uma empresa que tenta entrar em um mercado muito inovador sem comprovação de funcionalidade no mundo real, há mais chances de falha. Alguns fatores críticos de sucesso tem sido apontados como o processo de validação da ideia, que engloba falta de compreensão da essência dos mercados e dos concorrentes, timimg de implementação, equipe de sócios incapazes de lidar com as incertezas e suportar sua implicações (Ries, 2012; Blank \& Dorf, 2014; Cavalheiro, 2015; Reynolds 2016; Barnea, 2018; Ko \& An 2019)

As incertezas afetam quase todas as diferentes etapas do processo empreendedor, e o sucesso ou o fracasso das empresas depende de como os empreendedores lidam com as incertezas antes de agir em uma oportunidade (Rogers, 2011; Ries, 2012; Blank \& Dorf, 2014).

\section{Metodologia}

O presente artigo utiliza uma abordagem de pesquisa quantitativa, com o uso da técnica bibliométrica. Tal técnica mede os índices de produção e a disseminação do conhecimento científico (Araújo, 2006). Foi utilizada a lei da bibliometria de Bradford que avalia a concentração dos artigos nos periódicos sobre determinado assunto (Friedman, 2015). Foram utilizados os descritores fatores críticos de sucesso para a seleção da amostra de artigos. As fontes de coleta de dados foram as bases Google Acadêmico e Emerald por serem consideradas consolidadas e com acesso disponível. A amostra pesquisada totalizou 40 artigos, sendo selecionados por data (2014 a 2018) e por relevância.

\section{Análise dos resultados}

Quadro 1 - Objetivos e métodos dos mais relevantes (2014 a 2018)

\begin{tabular}{|l|l|l|l|}
\hline $\mathbf{N}^{\mathbf{0}}$ & \multicolumn{1}{|c|}{ Título } & Objetivo & Método \\
\hline 1 & $\begin{array}{l}\text { Acquisitions and Growth of } \\
\text { Software Startups: The Dual } \\
\text { Role of Venture Capital as a } \\
\text { Success Factor }\end{array}$ & $\begin{array}{l}\text { Investigar a interação entre os } \\
\text { investimentos em inovação na } \\
\text { pequena e grande empresa em um } \\
\text { contexto de inicialização. }\end{array}$ & Baseado em entrevistas estruturadas \\
\hline
\end{tabular}


2 Factors contributing to the early failure of small new charity start-ups
Estabelecer as principais causas de falha prematura de instituições de caridade de arrecadação de fundos recém-criada
A pesquisa combinou ideias da teoria do capital humano, ecologia organizacional e o princípio do sobrevivente para investigar a mortalidade de novas empresas de caridade.

3 Sustainable venture capital Fornecer uma visão de como os Painel de especialistas

e catalyst for sustainable capitalistas de risco podem start-up success? contribuir para o sucesso dos negócios sustentáveis.

$4 \quad$ Start-up Funding via Equity Analisar o conhecimento científico $\mathrm{O}$ estudo Crowdfunding in em relação aos fatores de sucesso Germany - A Qualitative do crowdfunding patrimonial. Analysis of Success Factors

5 Exploring entrepreneurial Analisar a

prontidão $\mathrm{O}$ estudo tem uma abordagem dedutiva readiness of youth and empreendedora dos jovens, hipotética. O estimador de máxima startup success components: principalmente em termos de verossimilhança foi utilizado como técnica Entrepreneurship training as capacidade empreendedora. a moderator de análise de dados através da modelagem de equações estruturais

$6 \quad$ Modelos de negócios à luz do lean startup: estudo de caso na itnc

Analisar o desenvolvimento de modelos de negócios baseados na metodologia Lean Startup.
7 Cross-sectional, qualitative Analisar os motivos das altas taxas in-depth interviews to de insucesso em empresas understand the success iniciantes, nos estágios iniciais de factors that Durban-based operação, em Durban, na África do start-up business owners $\mathrm{Sul}$ perceive as vital to grow from infancy to

8 Does personality of owners Examinar of micro enterprises matter efeito moderador dos Cinco Moderada (MMR) foi aplicada para for the relationship between Grandes fatores de personalidade teste para efeitos de previsão e moderação. startup capital and na relação entre capital inicial e entrepreneurial success? $\quad$ sucesso empresarial.

$9 \quad$ Coding skills as a success factor for a Society
Encontrar e classificar as diferentes Utilizou-se a revisão narrativa da literatura abordagens e métodos de promoção e aprendizagem de habilidades de codificação. 
10 Transforming entrepreneurial posture into a superior first product market position via dynamic capabilities and TMT prior start-up experience

\begin{tabular}{|c|c|}
\hline $\begin{array}{l}\text { Examinar o papel da exploração de } \\
\text { capacidades, experiência dos } \\
\text { diretores de start-up, colaboração } \\
\text { multifuncional e ativos de } \\
\text { tecnologia de informação } \mathrm{e} \\
\text { comunicação na condução da } \\
\text { postura empreendedora. }\end{array}$ & $\begin{array}{l}\text { Uma lista de NTVs foi adquirida através do } \\
\text { acesso a um diretório de empresas de um } \\
\text { departamento do governo na Índia. } \\
\text { Os NTVs amostrados tinham que ter } 3 \text { a } 5 \\
\text { anos de idade } \\
\text { Utilizamos a medida existente extraída da } \\
\text { literatura. }\end{array}$ \\
\hline
\end{tabular}

Fonte: dados da pesquisa

Nota: Obtida a partir do Google Acadêmico ${ }^{\circledR}$

A partir da análise do quadro 1, podemos verificar que existe relação entre os principais conceitos abordados no referencial teórico do presente trabalho, como por exemplo, empreendedorismo, lean startup, fatores de sucesso e modelo de negócio (Ries 2012; Sosna et al., 2010; Faltin \& Ripsas, 2011; Maurya, 2012 e Breuer 2013) com os artigos 1, 2, 4,5, 6,7 e 10 (Bennett 2016; Angerer, Brem, Kraus \& Peter 2017; Olugbola 2017; Powell 2017; Wagner 2017 e Ahmadi \& O'Cass 2018). E uma relação parcial com os artigos 3 e 8 (Bocken 2015; Baluku, Kikooma \& Kibanja 2016). Foi possível perceber ainda convergência entre o objetivo deste trabalho, que buscou identificar os principais fatores críticos de sucesso de uma startup e os artigos 4 e 7 e similaridade com os 1 e 3 . E por fim, observou-se uma discrepância de objetivo, com o artigo 9.

\section{Quadro 2 - Objetivos e métodos publicados em 2018}

\begin{tabular}{|c|c|c|c|}
\hline $\mathrm{N}^{\mathrm{o}}$ & Título & Objetivo & Método \\
\hline 1 & $\begin{array}{l}\text { Software engineering in } \\
\text { start-up companies: An } \\
\text { analysis of } 88 \text { experience } \\
\text { reports }\end{array}$ & $\begin{array}{l}\text { Investigar como a engenharia de } \\
\text { software é aplicada no contexto de } \\
\text { start-up. }\end{array}$ & $\begin{array}{l}\text { Realizamos um estudo exploratório } \\
\text { multivocal de } 88 \text { relatos de experiência } \\
\text { de start-up. }\end{array}$ \\
\hline 2 & $\begin{array}{l}\text { A startup cockpit for the } \\
\text { Proof-of-Concept }\end{array}$ & $\begin{array}{l}\text { Este artigo apresenta uma } \\
\text { ferramenta de medição - o Startup } \\
\text { Cockpit }\end{array}$ & $\begin{array}{l}\text { Foi realizada uma pesquisa } \\
\text { bibliográfica. }\end{array}$ \\
\hline 3 & $\begin{array}{l}\text { From Uncertainties to } \\
\text { Successful Start Ups: A Data } \\
\text { Analytic Approach to Predict } \\
\text { Success in Technological } \\
\text { Entrepreneurship }\end{array}$ & $\begin{array}{l}\text { Analisar e prever o sucesso futuro } \\
\text { de uma empresa, a partir da } \\
\text { identificação e análise das } \\
\text { incertezas que cercam as } \\
\text { oportunidades } \\
\text { estágio de avaliação de } \\
\text { oportunidade do processo } \\
\text { empreendedor. }\end{array}$ & $\begin{array}{l}\mathrm{Na} \text { primeira fase, os fatores de } \\
\text { incerteza são. Na segunda fase, um } \\
\text { modelo de previsão de sucesso é } \\
\text { implementado. usando técnicas de } \\
\text { aprendizado de máquina e } \\
\text { análise estratégica. }\end{array}$ \\
\hline 4 & $\begin{array}{l}\text { Lesson from Tokopedia. } \\
\text { com: E-commerce success } \\
\text { factor analysis: A case study } \\
\text { from Indonesian unicorn }\end{array}$ & $\begin{array}{l}\text { Quais os fatores que levaram o } \\
\text { Tokopedia ao sucesso }\end{array}$ & $\begin{array}{l}\text { A metodologia utilizada para } \\
\text { desenvolver este artigo foi o estudo de } \\
\text { caso. }\end{array}$ \\
\hline
\end{tabular}

DOI: $10.14211 / x i-e g e p e-118065$ 


\begin{tabular}{|c|c|c|c|}
\hline 5 & $\begin{array}{l}\text { High technology } \\
\text { entrepreneurs, their social } \\
\text { networks and success in } \\
\text { global markets: The case of } \\
\text { Israelis in the US market }\end{array}$ & $\begin{array}{l}\text { Analisar as relações entre redes } \\
\text { sociais, sucesso r de } \\
\text { empreendimentos de alta } \\
\text { tecnologia israelenses no mercado } \\
\text { dos EUA, atributos de rede e } \\
\text { padrões de utilização. }\end{array}$ & 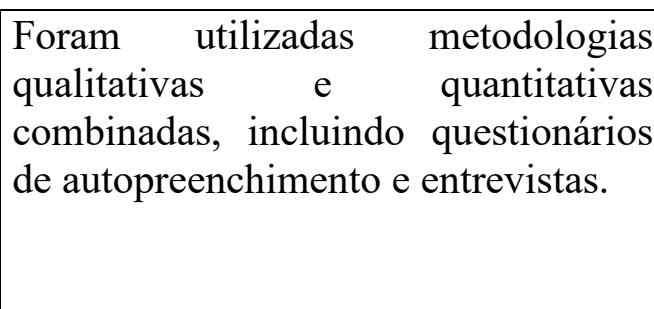 \\
\hline 6 & $\begin{array}{l}\text { Knowledge Flow, Innovative } \\
\text { Capabilities And Business } \\
\text { Success: Performance Of } \\
\text { The Relationship Between } \\
\text { Small World Networks To } \\
\text { Promote Innovation }\end{array}$ & $\begin{array}{l}\text { Analisar se as relações de rede } \\
\text { entre grupos étnicos semelhantes, } \\
\text { formação educacional e grupos } \\
\text { agrupados geograficamente } \\
\text { desempenham um papel } \\
\text { significativo na criação de } \\
\text { inovação ou não }\end{array}$ & $\begin{array}{l}\text { Utilizando o conceito teórico de uma } \\
\text { Small World Network, utilizamos } \\
\text { metodologia de estudo de caso para } \\
\text { estudar as origens de várias empresas } \\
\text { do setor de tecnologia, que nos últimos } \\
\text { anos surgiram como negócios de } \\
\text { sucesso }\end{array}$ \\
\hline 7 & $\begin{array}{l}\text { Critical Success Factors of a } \\
\text { Design Startup Business }\end{array}$ & $\begin{array}{l}\text { Determinar os fatores críticos de } \\
\text { sucesso que afetam as startups de } \\
\text { design. }\end{array}$ & Painel de especialista foi utilizado. \\
\hline 8 & $\begin{array}{l}\text { Comparison of selected } \\
\text { startup ecosystems in } \\
\text { Germany and in the USA } \\
\text { Explorative analysis of the } \\
\text { startup environments }\end{array}$ & $\begin{array}{l}\text { Este estudo objetiva analisar os } \\
\text { fatores críticos de sucesso na } \\
\text { Alemanha e EUA. }\end{array}$ & $\begin{array}{l}\text { A metodologia utilizada teve como } \\
\text { base pesquisa bibliográca e um painel } \\
\text { de especialistas. }\end{array}$ \\
\hline 9 & $\begin{array}{l}\text { The difficult path of startups } \\
\text { towards becoming middle- } \\
\text { size firms: the case of } \\
\text { entrepreneurial learning in } \\
\text { Québec's multimedia-IT } \\
\text { firms }\end{array}$ & $\begin{array}{l}\text { Analisar os fatores de sucesso que } \\
\text { levam ao crescimento das } \\
\text { pequenas e médias empresas, } \\
\text { inclusive startups, na região de } \\
\text { Quebec }\end{array}$ & $\begin{array}{l}\text { Revisão bibliográfica de relatórios } \\
\text { relevantes sobre empreendedorismo e } \\
\text { inovação e artigos científicos chave } \\
\text { relevantes. Em seguida, fizemos uma } \\
\text { análise temática e uma síntese dos } \\
\text { resultados. }\end{array}$ \\
\hline 10 & $\begin{array}{l}\text { The Moderating Role of } \\
\text { National Culture in the } \\
\text { Relationship Between } \\
\text { University entrepreneurship } \\
\text { Offerings and Student Start- } \\
\text { Up Activity: An } \\
\text { Embeddedness }\end{array}$ & $\begin{array}{l}\text { O artigo buscou analisar a } \\
\text { influência das práticas de } \\
\text { empreendedorismo } \\
\text { Universidade na iniciação de } \\
\text { negócios } \\
\end{array}$ & $\begin{array}{l}\text { Empregamos dados coletados através } \\
\text { do } 2011 \text { GUESSS1 para testar nossas } \\
\text { hipóteses. }\end{array}$ \\
\hline
\end{tabular}

Fonte: dados da pesquisa

Nota: Obtida a partir do Google Acadêmico ${ }^{\circledR}$

A partir da análise do quadro 2, podemos verificar que existe relação entre os principais conceitos abordados no referencial teórico do presente trabalho, como por exemplo, criação de negócios, startup e processo empreendedor, (Faltin \& Ripsas, 2011; Shane 2012; Ries 2012; Blank \& Dorf, 2014), com os artigos 3, 4, 5, 7, 89 e 10 (Dashti; Schwartz \& Pines 2018; Geibel \& Manickam 2018; Kim; Kim \& Jeon 2018; Kowanda; Firdaus; Pasaribu \& Nawangsari 2018; 
Rhéaume \& Tremblay 2018; e Shirokova; Tsukanova \& Morris 2018; Tomy \& Pardede 2018). E uma relação parcial com os artigos 3 e 4 (Kowanda, Firdaus, Pasaribu \& Nawangsari 2018; Tomy \& Pardede 2018). Foi possível perceber ainda convergência entre o objetivo deste trabalho que buscou identificar os fatores críticos de sucesso de uma startup e os artigos 3, 4, 7,8 e 9 e similaridade com os de número 2,5 e 10 . E por fim, observou-se uma discrepância de objetivo com o artigo 6.

\section{Quadro 3 - Objetivos e métodos dos mais relevantes}

\begin{tabular}{|c|c|c|c|}
\hline $\mathbf{N}^{\mathbf{o}}$ & Artigos & Objetivo & Método \\
\hline 1 & $\begin{array}{l}\text { Idea labs: instituting an } \\
\text { innovation discovery } \\
\text { process capable of } \\
\text { sustaining the business }\end{array}$ & 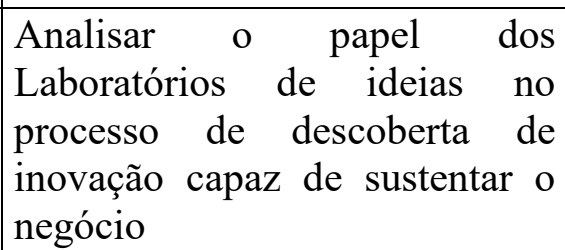 & Pesquisa bibliográfica \\
\hline 2 & $\begin{array}{l}\text { Dedicated } \\
\text { centers in pusiness } \\
\text { libraries }\end{array}$ & $\begin{array}{l}\text { Determinar se um centro de } \\
\text { negócios dedicado ou não dentro } \\
\text { de uma biblioteca pública atua } \\
\text { como um fator-chave de } \\
\text { sucesso. }\end{array}$ & $\begin{array}{l}\text { Uma pesquisa com questionários foi } \\
\text { enviada para } 88 \text { bibliotecas públicas com } \\
\text { centros de negócios dedicados, e postadas } \\
\text { no BRASS-L e BUSLIB-. }\end{array}$ \\
\hline 3 & $\begin{array}{l}\text { New educational } \\
\text { services development: } \\
\text { Framework for } \\
\text { technology } \\
\text { entrepreneurship at } \\
\text { education } \\
\text { universities in Egypt }\end{array}$ & $\begin{array}{l}\text { O objetivo deste artigo é } \\
\text { desenvolver uma estrutura para a } \\
\text { educação em empreendedorismo } \\
\text { tecnológico (TEPE) dentro das } \\
\text { universidades. }\end{array}$ & $\begin{array}{l}\text { Abordagens de pesquisa qualitativa e } \\
\text { quantitativa foram empregadas. }\end{array}$ \\
\hline 4 & $\begin{array}{l}\text { How emerging market } \\
\text { resource-poor firms } \\
\text { competer and } \\
\text { outcompete advanced } \\
\text { country resource-rich } \\
\text { rivals: An asymmetry } \\
\text { reversing theory }\end{array}$ & $\begin{array}{l}\text { Comentar a recente publicação } \\
\text { do professor Ming-Jer Chen } \\
\text { intitulada "Dinâmica } \\
\text { competitiva: raízes orientais, } \\
\text { crescimento ocidental" e } \\
\text { apresentar uma perspectiva de } \\
\text { reversão da assimetria na } \\
\text { dinâmica competitiva entre dois } \\
\text { concorrentes não óbvios, } \\
\text { invisíveis ou indiretos. }\end{array}$ & $\begin{array}{l}\text { O autor primeiro identifica uma } \\
\text { negligência importante na pesquisa do } \\
\text { professor Chen sobre a dinâmica } \\
\text { competitiva. Em seguida, o autor propõe } \\
\text { uma teoria de reversão de assimetria (ART) } \\
\text { da dinâmica competitiva para corrigir essa } \\
\text { negligência. }\end{array}$ \\
\hline 5 & $\begin{array}{l}\text { Enacting the lean } \\
\text { startup methodology: } \\
\text { The role of vicarious } \\
\text { and experiential } \\
\text { learning processes }\end{array}$ & $\begin{array}{l}\text { O objetivo deste artigo é } \\
\text { examinar como as instruções da } \\
\text { metodologia lean startup } \\
\text { influenciam os empreendedores }\end{array}$ & $\begin{array}{l}\text { Este trabalho emprega um projeto de } \\
\text { entrevista } \\
\text { fenomenológica em profundidade. }\end{array}$ \\
\hline
\end{tabular}




\begin{tabular}{|c|c|c|c|}
\hline 6 & $\begin{array}{l}\text { Configurations of new } \\
\text { ventures in } \\
\text { entrepreneurship } \\
\text { research: contributions } \\
\text { and research gaps }\end{array}$ & $\begin{array}{l}\text { Fornecer uma visão geral } \\
\text { estruturada da aplicação da } \\
\text { abordagem de configuração no } \\
\text { contexto de novos e pequenos } \\
\text { empreendimentos. }\end{array}$ & $\begin{array}{l}\text { O artigo examina a literatura empírica nos } \\
\text { principais periódicos de } \\
\text { empreendedorismo de } 1994 \text { a } 2006 .\end{array}$ \\
\hline 7 & $\begin{array}{l}\text { An Investigation of } \\
\text { Centers for Innovation }\end{array}$ & \begin{tabular}{|lrrr}
$\begin{array}{l}\text { Esclarecer } \\
\text { organizações }\end{array}$ & $\begin{array}{r}\text { por } \\
\text { em }\end{array}$ & que as \\
vários \\
$\begin{array}{lll}\text { contextos estão } & \text { buscando } \\
\text { centros de inovação. }\end{array}$
\end{tabular} & $\begin{array}{l}\text { Examinando as declarações de missão } \\
\text { seguidas por um levantamento eletrônico } \\
\text { de } 66 \text { centros de inovação, caracterizamos } \\
\text { as práticas, fundamentos, fatores de } \\
\text { sucesso, desafios e outros descritores } \\
\text { desses centros. }\end{array}$ \\
\hline 8 & $\begin{array}{l}\text { Exploring } \\
\text { entrepreneurial } \\
\text { conceptions, beliefs } \\
\text { and intentions of Greek } \\
\text { graduates }\end{array}$ & $\begin{array}{l}\text { Identificar concepções } \\
\text { empreendedoras e crenças de } \\
\text { graduados gregos, comparando } \\
\text { graduados em ciência e } \\
\text { economia e empreendedores da } \\
\text { população em geral. }\end{array}$ & $\begin{array}{l}\text { O modelo de equações estruturais é usado } \\
\text { para recuperar um padrão explicativo para } \\
\text { as variáveis e dados presentes. }\end{array}$ \\
\hline 9 & $\begin{array}{l}\text { The enterprising } \\
\text { communities and } \\
\text { startup ecosystem in } \\
\text { Iran }\end{array}$ & $\begin{array}{l}\text { Concentra-se no conceito de } \\
\text { "startups" e tenta apresentar uma } \\
\text { visão mais abrangente deste } \\
\text { fenômeno.. }\end{array}$ & $\begin{array}{l}\text { O autor elabora os estágios e desafios } \\
\text { existentes das startups no Irã. Para isso, } 65 \\
\text { fundadores de startups de tecnologia são } \\
\text { entrevistados. }\end{array}$ \\
\hline 10 & $\begin{array}{l}\text { High-tech } \\
\text { entrepreneurial content } \\
\text { marketing for business } \\
\text { model innovation: A } \\
\text { conceptual framework }\end{array}$ & $\begin{array}{l}\text { Promover o desenvolvimento } \\
\text { teórico do conceito de marketing } \\
\text { de conteúdo e sua integração à } \\
\text { teoria de marketing de alta } \\
\text { tecnologia, em contextos } \\
\text { empreendedores e a partir de } \\
\text { uma perspectiva de inovação de } \\
\text { modelos de negócios. }\end{array}$ & $\begin{array}{l}\text { The paper provides a conceptual overview } \\
\text { of content marketing and business model } \\
\text { innovation concerning high-tech } \\
\text { entrepreneurs. }\end{array}$ \\
\hline
\end{tabular}

Fonte: dados da pesquisa

Nota: Obtida a partir da Emerald $\AA$

A partir da análise do quadro 3, podemos verificar que existe relação entre os principais conceitos abordados no referencial teórico do presente trabalho, como por exemplo, empreendedores, inovação e startup (Ries, 2012; Blank e Dorf, 2014; Cavalheiro, 2015; Barnea, 2018) com os artigos 3; 7, 8, 9 e 10 (Abou-Warda 2016; Mansour \& Barandas 2017; Salamzadeh \& Kawamorita 2017 e Kahn \& Kakouris, 2016). E uma relação parcial com os artigos 1 e 6 (Harms, Kraus \& Reschke 2007; Narayanan 2017). Foi possível perceber ainda convergência entre o objetivo deste trabalho e o artigo 9 (Salamzadeh \& Kawamorita 2017) e similaridade com os 1, 2, 3, 7, 8 e 10. E por fim, observou-se uma discrepância de objetivo com os artigos 4,5 e 7. 
Quadro 4 - Objetivos e métodos publicados em 2018

\begin{tabular}{|c|c|c|c|}
\hline $\mathbf{N}^{\mathbf{o}}$ & Artigos & Objetivo & Método \\
\hline 1 & $\begin{array}{l}\text { Enhancing } \\
\text { sustainable urban } \\
\text { development } \\
\text { through smart city } \\
\text { applications }\end{array}$ & $\begin{array}{l}\text { Este artigo investiga a contribuição } \\
\text { potencial de abordagens e } \\
\text { ferramentas de cidades inteligentes } \\
\text { para o desenvolvimento urbano } \\
\text { sustentável no domínio do meio } \\
\text { ambiente. }\end{array}$ & $\begin{array}{l}\text { Este objetivo é perseguido através de uma } \\
\text { investigação aprofundada das aplicações de } \\
\text { código aberto disponíveis e proprietárias de } \\
\text { cidades inteligentes relacionadas à } \\
\text { sustentabilidade ambiental em ambientes } \\
\text { urbanos. }\end{array}$ \\
\hline 2 & $\begin{array}{l}\text { Studying the } \\
\text { relationship between } \\
\text { the perceived value } \\
\text { of online group- } \\
\text { buying websites and } \\
\text { customer loyalty: } \\
\text { the moderating role } \\
\text { of referral rewards }\end{array}$ & $\begin{array}{l}\text { Examinar o efeito do valor } \\
\text { percebido na fidelidade do cliente } \\
\text { por meio do comprometimento } \\
\text { afetivo no contexto de compra em } \\
\text { grupo (OGB) online. }\end{array}$ & $\begin{array}{l}\text { Todos os dados foram coletados dos membros } \\
\text { dos sites da OGB em Taiwan. O número total } \\
\text { de entrevistados na pesquisa on-line foi } 403 \text {. Os } \\
\text { dados foram analisados usando modelagem de } \\
\text { equações estruturais (SEM) para testar um } \\
\text { modelo de valor percebido - comprometimento } \\
\text { - lealdade. }\end{array}$ \\
\hline 3 & $\begin{array}{l}\text { Modelling the } \\
\text { critical success } \\
\text { factors of women } \\
\text { entrepreneurship } \\
\text { using fuzzy AHP } \\
\text { framework }\end{array}$ & $\begin{array}{l}\text { Identificar, priorizar e avaliar os } \\
\text { fatores críticos de sucesso da } \\
\text { adoção do empreendedorismo } \\
\text { feminino nas MPMEs indianas. }\end{array}$ & $\begin{array}{l}\text { Este artigo propõe uma metodologia baseada no } \\
\text { processo hierárquico analítico difuso para } \\
\text { priorizar os fatores críticos de sucesso da } \\
\text { adoção do empreendedorismo feminino. }\end{array}$ \\
\hline 4 & $\begin{array}{l}\text { Culture and social } \\
\text { capital network } \\
\text { effects on the } \\
\text { survival } \\
\text { performance and } \\
\text { high-tech micro and } \\
\text { small firms }\end{array}$ & $\begin{array}{l}\text { Propor uma teoria integrada para } \\
\text { explicar o efeito da cultura regional } \\
\text { sobre os resultados das empresas de } \\
\text { alta tecnologia micro e pequena }\end{array}$ & $\begin{array}{l}\text { Os insights teóricos do capital social e da } \\
\text { cultura são combinados com os resultados das } \\
\text { observações empíricas anteriores para explicar } \\
\text { as diferenças transculturais no desempenho das } \\
\text { empresas HTMS. }\end{array}$ \\
\hline 5 & $\begin{array}{l}\text { A TISM modeling } \\
\text { of critical success } \\
\text { factors r of } \\
\text { blockchain based } \\
\text { cloud services }\end{array}$ & $\begin{array}{l}\text { Identificar, analisar e compreender } \\
\text { as interações mútuas entre os vários } \\
\text { fatores críticos de sucesso (CSFs) } \\
\text { que podem facilitar o sucesso de } \\
\text { serviços em nuvem baseados em } \\
\text { blockchain. }\end{array}$ & $\begin{array}{l}\text { Neste trabalho, } 19 \text { FCs foram identificados } \\
\text { através de revisão de literatura e opiniões de } \\
\text { especialistas. }\end{array}$ \\
\hline
\end{tabular}




\begin{tabular}{|c|c|c|c|}
\hline 6 & $\begin{array}{lr}\text { Case study } & \text { on } \\
\text { adoption of new } \\
\text { technology } & \text { for } \\
\text { innovation: } & \\
\text { Perspective } & \text { of } \\
\text { institutional and } \\
\text { corporate } \\
\text { entrepreneurship }\end{array}$ & $\begin{array}{l}\text { Investigar o papel do } \\
\text { empreendedorismo institucional e } \\
\text { empreendedorismo corporativo. } \\
\text { para lidar com os impasses da } \\
\text { empresa, adotando a nova } \\
\text { tecnologia à frente de outras } \\
\text { empresas. }\end{array}$ & $\begin{array}{l}\text { O quadro de pesquisa utilizado é o seguinte: } \\
\text { primeiro, a perspectiva dos estudos sobre } \\
\text { empreendedorismo institucional e corporativo é } \\
\text { realizada usando literatura prévia e referências } \\
\text { preliminares; segundo, foi proposto um quadro } \\
\text { analítico de pesquisa; finalmente, os casos } \\
\text { baseados em fases são conduzidos de forma a } \\
\text { identificar o objetivo da pesquisa. }\end{array}$ \\
\hline 7 & $\begin{array}{l}\text { University-based } \\
\text { incubators' } \\
\text { performance } \\
\text { evaluation: } \\
\text { benchmarking } \\
\text { approach }\end{array}$ & $\begin{array}{l}\text { Entender as práticas de incubação } \\
\text { existentes e desenvolver sugestões } \\
\text { desenvolvendo um estudo } \\
\text { comparativo com as incubadoras de } \\
\text { universidades de Taiwan }\end{array}$ & $\begin{array}{l}\text { Este resultado de pesquisa é baseado na } \\
\text { metodologia para gerar um benchmarking a } \\
\text { partir de seis UBIs de Taiwan e para outras } \\
\text { incubadoras de empresas em todo o mundo. }\end{array}$ \\
\hline 8 & $\begin{array}{l}\text { What does it take to } \\
\text { implement open } \\
\text { innovation? } \\
\text { Towards an } \\
\text { integrated capability } \\
\text { framework }\end{array}$ & $\begin{array}{l}\text { Como a mudança para a inovação } \\
\text { aberta (OI) é difícil e faz com que } \\
\text { muitas iniciativas falhem, surge a } \\
\text { questão de quais capacidades as } \\
\text { organizações devem desenvolver } \\
\text { para implementar com sucesso a } \\
\text { OI. Como a literatura abrange } \\
\text { fluxos maduros, mas isolados, em } \\
\text { capacidades de OI, existe a } \\
\text { necessidade de uma estrutura de } \\
\text { capacidade integrada. O artigo } \\
\text { pretende discutir essas questões. }\end{array}$ & $\begin{array}{l}\text { Este artigo propõe a estrutura de capacidade de } \\
\text { inovação aberta (OICF) que compila e estrutura } \\
\text { as capacidades relevantes para a } \\
\text { implementação da OI. O OICF cobre os } \\
\text { processos de OI externos e acoplados. Para } \\
\text { integrar múltiplos fluxos da literatura OI, o } \\
\text { OICF baseia-se em uma revisão estruturada da } \\
\text { literatura. O OICF também foi validado em um } \\
\text { processo de revisão de duas etapas com } \\
\text { especialistas da OI da academia e da indústria. }\end{array}$ \\
\hline 9 & $\begin{array}{l}\text { Strategies and } \\
\text { service innovations } \\
\text { of haitao business in } \\
\text { the Chinese market: } \\
\text { A comparative case } \\
\text { study of Amazon.cn } \\
\text { vs Gmarket.co.kr }\end{array}$ & $\begin{array}{l}\text { Este estudo tem como objetivo } \\
\text { explorar as estratégias de inovação } \\
\text { de serviços e os fatores de sucesso } \\
\text { dos negócios haitao no mercado } \\
\text { chinês. }\end{array}$ & $\begin{array}{l}\text { Os autores selecionaram dois sites haitao de } \\
\text { sucesso da Amazon.cn e Gmarket.co.kr, como } \\
\text { representantes de um player global e um player } \\
\text { de nicho, e conduziram um estudo de caso } \\
\text { comparativo para analisar suas estratégias de } \\
\text { inovação de serviços e os principais fatores de } \\
\text { sucesso. }\end{array}$ \\
\hline 10 & $\begin{array}{l}\text { Generating } \\
\text { resources through } \\
\text { co-evolution of } \\
\text { entrepreneurs and } \\
\text { ecosystems }\end{array}$ & $\begin{array}{l}\text { Este trabalho tem como objetivo } \\
\text { estudar a interação entre o processo } \\
\text { de construção de oportunidades e o } \\
\text { desenvolvimento de recursos no } \\
\text { ecossistema circundante. }\end{array}$ & $\begin{array}{l}\text { Este artigo é uma análise qualitativa do caso } \\
\text { extremo da Aalto Entrepreneurship Society } \\
\text { (Aaltoes), uma organização recém-fundada que } \\
\text { promove com base em entrevistas de seis } \\
\text { colaboradores principais e quatro organizações } \\
\text { de partes interessadas. }\end{array}$ \\
\hline
\end{tabular}

Fonte: dados da pesquisa

Nota: Obtida a partir da Emerald ${ }^{\circledR}$ 
A partir da análise do quadro 4, podemos verificar que existe relação entre os principais conceitos abordados no referencial teórico do presente trabalho, como por exemplo, empreendedorismo, startup e fatores críticos de sucesso (Ries, 2012; Blank e Dorf 2014; Liu \& Hong 2016; Geibel \& Manickam 2018; Kim; Kim; \& Jeon, 2018; Kowanda; Firdaus; Pasaribu, \& Nawangsari, 2018; Prasad; Shankar \& Roy 2018; Ripsas; Schaper \& Tröger, 2018; Rhéaume, \& Tremblay 2018; Tomy; \& Pardede, 2018) com os artigos 3, 4 e 9 (Liu, C., \& Hong, J. (2016); Amrita, Garg \& Singh 2018; Hallam, C; Dosamantes, C. A.D \& Zanella, G. 2018). E uma relação parcial com os artigos $2,5,6,7,8$ e 10. É possível perceber ainda convergência entre o objetivo deste trabalho e os artigos 3, 5 e 9 (Liu, C., \& Hong, J. (2016; Amrita; Garg \& Singh 2018; Prasad; Shankar; Gupta \& Roy; 2018) e similaridade com os 2, 6 e 10. E por fim, observou-se uma discrepância de objetivo com o artigo 1.

\section{Conclusões}

O presente artigo objetivou identificar os principais fatores críticos de sucesso de uma Startup. Para tanto, foram utilizadas base de dados que contempla artigos internacionais como fonte de coleta de dados. As bases utilizadas foram o Google Acadêmico e Emerald. Os descritores utilizados foram fatores críticos de sucesso, totalizando uma amostra de 40 artigos, selecionados por data e relevância.

Os resultados indicam que existe similaridade entre os objetivos da amostra com os do presente artigo, evidenciando a importância do tema. Os artigos da amostra mostraram ainda uma convergência com este trabalho, no que concerne aos conceitos relativos a criação de negócios e principais definições de startups.

Escalar prematuramente, processo de validação da ideia, timimg de implementação e equipe de sócios incapazes de lidar com as incertezas e suportar suas implicações, foram identificados como os principais fatores críticos de sucesso das startups, indicando que futuros empreendedores precisam dar atenção especial a eles.

O principal avanço que este artigo promoveu foi, a partir de (Liu \& Hong 2016; Angerer, Brem, Kraus \& Peter 2017; Powell, 2017; Salamzadeh \& Kawamorita 2017; Amrita, Garg \& Singh 2018; Geibel \& Manickam 2018; Kim, Kim \& Jeon 2018; Kowanda, Firdaus, Pasaribu \& Nawangsari 2018; Prasad; Shankar; Gupta \& Roy; 2018; Rhéaume \& Tremblay 2018 e Tomy $\&$ Pardede 2018), apresentar os principais fatores críticos de sucesso de uma startup.

Este artigo contribui ainda com uma reflexão sobre os impactos das altas taxas de mortalidade de startups (Behrens, 2015; Patell, 2015) na taxa de desemprego e crescimento da economia.

A limitação desta pesquisa foi a escolha da amostra, baseada nos indexadores dos mescanismos de busca Google Acadêmico e Emerald.

Sugere-se para estudos futuros investigar os fatores críticos de sucesso sob a ótica de investidores anjo e fundos de venture capital. E ainda uma análise sobre os impactos das altas taxas de mortalidade de startups no índice de desemprego e crescimento da economia. 


\section{Referências}

Abou-Warda, S. H. (2016). New educational services development: framework for technology entrepreneurship education at universities in Egypt. International Journal of Educational Management, 30(5), 698-717.

Ahmadi, H., \& O'Cass, A. (2018). Transforming entrepreneurial posture into a superior first product market position via dynamic capabilities and TMT prior start-up experience. Industrial Marketing Management, 68, 95-105.

Amrita, K., Garg, C. P., \& Singh, S. (2018). Modelling the critical success factors of women entrepreneurship using fuzzy AHP framework. Journal of Entrepreneurship in Emerging Economies, 10(1), 81-116.

Angerer, M., Brem, A., Kraus, S., \& Peter, A. (2017). Start-up funding via equity crowdfunding in germany-a qualitative analysis of success factors. The Journal of Entrepreneurial Finance, 19(1), 1 .

Araujo, C. A. Bibliometria: evolução histórica e questões atuais. Em Questão. Porto Alegre, v. 12, n. 1, jan/jun, p. 11-32, 2006.

Bakator, M., Đorđević, D., Ćoćkalo, D., Nikolić, M., \& Vorkapić, M. (2018). Lean startups with industry 4.0 technologies: Overcoming the challenges of youth entrepreneurship in Serbia. Journal of Engineering Management and Competitiveness (JEMC), 8(2), 89-101

Barnea, A. (2018). Israeli start-ups-especially in cyber security: Can a new model enhance their survival rate?. Journal of Intelligence Studies in Business, 8(1).

Baluku, M. M., Kikooma, J. F., \& Kibanja, G. M. (2016). Does personality of owners of micro enterprises matter for the relationship between startup capital and entrepreneurial success?. African Journal of Business Management, 10(1), 13-23.

Behrens, J. S. B. (2015). Startup na prática: desafios e oportunidades.

Bennett, R. (2016). Factors contributing to the early failure of small new charity startups. Journal of Small Business and Enterprise Development, 23(2), 333-348.

Blank, s. \& Dorf, b. - "Startup: Entrepreneur's Manual" Step-by-Step Guide to Building a Large Enterprise. Alta Books 2014

Bocken, N. M. (2015). Sustainable venture capital-catalyst for sustainable start-up success?. Journal of Cleaner Production, 108, 647-658.

Breuer, H. (2013) Lean venturing: learning to create new business through exploration, elaboration, evaluation, experimentation, and evolution. International Journal of Innovation Management, 17(03): 1340013

Cavalheiro, Cristian Mairesse. Fatores determinantes para o sucesso de startups de TI no Brasil: uma avaliação crítica. 2015. 198 f. Dissertação (Mestrado) - Curso de Gestão e Negócio, Unidade Acadêmica de Pesquisa e Pós-graduação, Universidade do Vale do Rio dos Sinos Unisinos, Porto Alegre. D 
Dashti, Y., Schwartz, D., \& Pines, A. M. (2018). High technology entrepreneurs, their social networks and success in global markets: The case of Israelis in the US market. Current Topics in Management: Volume 13, Global Perspectives on Strategy, Behavior, and Performance, 131.

Faltin, G., \& Ripsas, S. (2011). Das Gestalten von Geschäftsmodellen als Kern des Entrepreneurship (No. 61). Working Papers of the Institute of Management Berlin at the Berlin School of Economics and Law (HWR Berlin).

Friedman, A. (2015). Lotka's Law package.

Geibel, R. C., \& Manickam, M. (2018). Comparison of selected startup ecosystems in Germany and in the USA Explorative analysis of the startup environments. GSTF Journal on Business Review (GBR), 4(3).

Gielnik, M. M., Zacher, H., \& Wang, M. (2018). Age in the entrepreneurial process: The role of future time perspective and prior entrepreneurial experience. Journal of Applied Psychology, 103(10), 1067

Hallam,C; Dosamantes, C. A. D \& Zanella, G. (2018) "Culture and social capital network effects on the survival and performance of high-tech micro and small firms", Journal of Small Business and Enterprise Development, Vol. 25 Issue: 1, pp.81-106, .

Harms, R., Kraus, S., \& Reschke, C. H. (2007). Configurations of new ventures in entrepreneurship research: contributions and research gaps. Management Research News, 30(9), 661-673.

Kahn, K \& Dempsey, J (2012) "An Investigation of Centers for Innovation", International Journal of Innovation Science, Vol. 4 Issue: 2, pp.89-100, https://doi.org/10.1260/1757$\underline{2223.4 .2 .89}$.

Kakouris, A (2016) "Exploring entrepreneurial conceptions, beliefs and intentions of Greek graduates", International Journal of Entrepreneurial Behavior \& Research, Vol. 22 Issue: 1, pp.109-132, https://doi.org/10.1108/IJEBR-07-2014-013 ART 8 QD 3

Kim, B., Kim, H., \& Jeon, Y. (2018). Critical Success Factors of a Design Startup Business. Sustainability, 10(9), 2981

Klotins, E., Unterkalmsteiner, M., \& Gorschek, T. (2019). Software engineering in start-up companies: An analysis of 88 experience reports. Empirical Software Engineering, 24(1), 68102.

Ko, C. R., \& An, J. I. (2019). Success Factors of Student Startups in Korea: From Employment Measures to Market Success. Asian Journal of Innovation \& Policy, 8(1).

Kowanda, D., Firdaus, M., Pasaribu, R. B. F., \& Nawangsari, S. (2018, January). Lesson from Tokopedia. com: E-commerce success factor analysis: A case study from Indonesian unicorn. 
In 2018 International Conference on Information Management and Processing (ICIMP) (pp. 61-65). IEEE.

Kohler, T. (2016). Corporate accelerators: Building bridges between corporations and startups. Business Horizons, 59(3), 347-357.

Liu, C., \& Hong, J. (2016). Strategies and service innovations of haitao business in the Chinese market: A comparative case study of Amazon. cn vs Gmarket. co. kr. Asia Pacific Journal of Innovation and Entrepreneurship, 10(1), 101-121.

Mansour, D., \& Barandas, H. (2017). High-tech entrepreneurial content marketing for business model innovation: A conceptual framework. Journal of Research in Interactive Marketing, 11(3), 296-311.

Maurya, A. (2012) Running Lean: Iterate from Plan A to a Plan That Works. O’Reilly Media.

Mcclelland, David. A sociedade competitiva: realização e progresso social. Rio de Janeiro: Expressão e Cultura, 1972.

McCann, B. T., \& Vroo)m, G. (2015. Opportunity evaluation and changing beliefs during the nascent entrepreneurial process. International small business journal, 33(6), 612-637.

Meyer, J. Welcome to entrepreneur country. London: Constable, 2012

Narayanan, V. K. (2017). Idea labs: instituting an innovation discovery process capable of sustaining the business. Strategy \& Leadership, 45(1), 27-36.

Olugbola, S. A. (2017). Exploring entrepreneurial readiness of youth and startup success components: Entrepreneurship training as a moderator. Journal of Innovation \& Knowledge, 2(3), 155-171.

Packard, M. D., Clark, B. B., \& Klein, P. G. (2017). Uncertainty types and transitions in the entrepreneurial process. Organization Science, 28(5), 840-856

Patel, N. (2018). 90\% of startups fail: Here's what you need to know about the 10\%. 2015.

Powell, D. (2017). Cross-sectional, qualitative in-depth interviews to understand the success factors that Durban-based start-up business owners perceive as vital to grow from infancy to adolescence in the organisational life cycle, using the Concept of Key Success Factors by Grunert and Ellegaard (1992) (Doctoral dissertation, The IIE).

Prasad, S., Shankar, R., Gupta, R., \& Roy, S. (2018). A TISM modeling of critical success factors of blockchain based cloud services. Journal of Advances in Management Research, 15(4), 434-456.

Radu-Daniel, P., Daniel, P., Cristian, D., Irina, S., \& Daniela-Rodica, S. (2015). Structural Changes in the entrepreneurial profile of the creative industries in Romania. Procedia Economics and Finance, 23, 1147-1151

Reynolds, P. D. (2016). Start-up actions and outcomes: What entrepreneurs do to reach profitability. Foundations and Trends ${ }^{\circledR}$ in Entrepreneurship, 12(6), 443-559. 
Rhéaume, L., \& Tremblay, D. G. (2018). The difficult path of startups towards becoming middle-size firms: the case of entrepreneurial learning in Québec's multimedia-IT firms.

Ries, E. (2012). A startup enxuta. São Paulo: Leya

Ripsas, S., Schaper, B., \& Tröger, S. (2018). A startup cockpit for the proof-ofconcept. Handbuch Entrepreneurship, 1-17.

Rogers, S. Finanças e Estratégias de Negócios para Empreendedores. Porto Alegre: Bookman, 2011.

Salamzadeh, A., \& Kawamorita Kesim, H. (2017). The enterprising communities and startup ecosystem in Iran. Journal of Enterprising Communities: People and Places in the Global Economy, 11(4), 456-479..

Schmidt, s.; Bohnenberger, M. C. Perfil empreendedor e desempenho organizacional. Revista de Administração Contemporânea, Rio de Janeiro, v. 13, p. 450-467, 2009

Shane, S. (2012). Reflections on the 2010 AMR decade award: delivering on the promise of entrepreneurship as a field of research. Academy of Management Review, 37(1), 10-20. doi: 10.5465/amr.2011.0078

Shirokova, G., Tsukanova, T., \& Morris, M. H. (2018). The moderating role of national culture in the relationship between university entrepreneurship offerings and student start-up activity: an embeddedness perspective. Journal of Small Business Management, 56(1), 103-130.

Sivapalan, A., \& Balasundaram, N. (2012). Level of entrepreneurial intention of the management undergraduates in the University of Jaffna, Sri Lanka: scholars and undergraduates perspective. South Asian Academic Research Journals, 2(10),24-42.

Sosna, M., Trevinyo-Rodríguez, R.N. \& Velamuri, S.R. (2010) Business Model Innovation through Trial-and-Error Learning: The Naturhouse Case. Long Range Planning

Tomy, S., \& Pardede, E. (2018). From uncertainties to successful start ups: A data analytic approach to predict success in technological entrepreneurship. Sustainability, 10(3), 602.

Ummah, S., \& Gunapalan, S. (2012). Factors influencing on entrepreneurial success: an empirical study on women headed families in Ampara and Batticaloa districts in Sri Lanka. International Journal of Business, Economics and Law, 1(1),141-148

Wagner, M. (2017, June). Acquisitions and Growth of Software Startups: The Dual Role of Venture Capital as a Success Factor. In International Conference of Software Business (pp. 1831). Springer, Cham.

https://www1.folha.uol.com.br/mercado/2018/03/investimento-em-startups-brasileiras-baterecorde-em-2017.shtml 